\title{
Recent BaBar results in charmonium and charm spectroscopy
}

\author{
Enrico Robutti, representing the $B A B A R$ Collaboration \\ I.N.F.N., Sezione di Genova, Via Dodecaneso 33, 16146 Genova, Italy
}

Received: / Revised version:

\begin{abstract}
Recent results are presented on the study of the $\eta_{c}$ and $\eta_{c}(2 S)$ resonance parameters and on the observation of two narrow states in the $D_{s}$ spectrum, using the BABAR data set. The $\eta_{c}$ and $\eta_{c}(2 S)$ are produced in $\gamma \gamma$ interactions: a precision measurement of their mass and total width is reported, together with a study supporting the correct assignment of the $\eta_{c}(2 S)$ quantum numbers. Two states $D_{s . J}^{*+} 2317$, $D_{s J 2457}^{+}$are observed in continuum $e^{+} e^{-}$production, decaying to $D_{s}^{+} \pi^{0}$ and $D_{s}^{+} \pi^{0} \gamma$ respectively: their masses are measured and a study on the resonant structure of the $D_{s J}^{+}(2457)$ decay is reported. All results are preliminary ${ }^{\mathrm{a}}$.
\end{abstract}

PACS. PACS-13.66.Bc - PACS-14.40.Gx - PACS-14.40.Lb

\section{Measurement of the $\eta_{c}$ and $\eta_{c}(2 S)$ resonance parameters}

The mass and width of the $\eta_{c}$ meson, the lowest lying state of charmonium, are not well established after almost 30 years from its first observation. In particular, the most recent measurements of the the total width [2] point to values substantially larger than the current world average [3]. The first radial excitation of the $\eta_{c}$, the $\eta_{c}(2 S)$, has recently been observed by Belle in exclusive $B^{+} \rightarrow$ $\eta_{c}(2 S) K^{+}, \eta_{c}(2 S) \rightarrow K_{S}^{0} K^{+} \pi^{-}$decays [4] [5] and in the $J / \psi$ recoil spectrum in $e^{+} e^{-}$annihilations [6], at a mass significantly higher than what originally reported by the Crystal Ball experiment [7].

The $\eta_{c}$ is known to be coupled to the $\gamma \gamma$ channel $\left(\mathcal{B}\left(\eta_{c} \rightarrow \gamma \gamma\right) \sim 2 \cdot 10^{-4}\right)$. At $B A B A R, \gamma \gamma$ interactions result from virtual photons emitted by electrons and positrons in the colliding beams: the cross section is highly peaked at small angles, so that in most cases the electron and positron remain undetected and the momentum of detected particles is balanced in the transverse plane.

In this analysis, $K_{S}^{0} K^{+} \pi^{-}$final states are considered. Events are selected by requiring four charged particles with total transverse momentum $p_{\mathrm{T}}<0.5 \mathrm{GeV} / c$ and total energy in the laboratory frame $E_{\text {tot }}>9 \mathrm{GeV}$, in order to suppress continuum $e^{+} e^{-} \rightarrow q \bar{q}$ events. $K_{S}^{0}$ are reconstructed in the $\pi^{+} \pi^{-}$decay mode, and particle identification criteria are applied to $K^{ \pm}$candidates. A common vertex is required.

Figure 1 (a) shows the resulting $K_{S}^{0} K^{+} \pi^{-}$invariant mass spectrum. The presence of a peak at the $J / \psi$ mass is due to initial state radiation events, where a photon is emitted in the initial state, and a backward-going $J / \psi$ is produced, its decay products falling into the detector

a Except for the of $D_{s J}^{*}(2317)$ mass determination. acceptance because of the Lorentz boost of the center of mass. A fit to this distribution to a sum of a smooth background shape, a Gaussian function for the $J / \psi$ peak and the convolution of a non-relativistic Breit-Wigner shape with a Gaussian resolution function for the $\eta_{c}$ peak, yields

$$
\begin{gathered}
m\left(\eta_{c}\right)=(2983.3 \pm 1.2(\text { stat }) \pm 1.8(\text { syst })) \mathrm{MeV} / c^{2} \\
\Gamma\left(\eta_{c}\right)=(33.3 \pm 2.5(\text { stat }) \pm 0.8(\text { stat })) \mathrm{MeV} / c^{2}
\end{gathered}
$$

The resolution is determined from the $J / \psi$ peak; a correction is applied to the mass scale to account for a bias

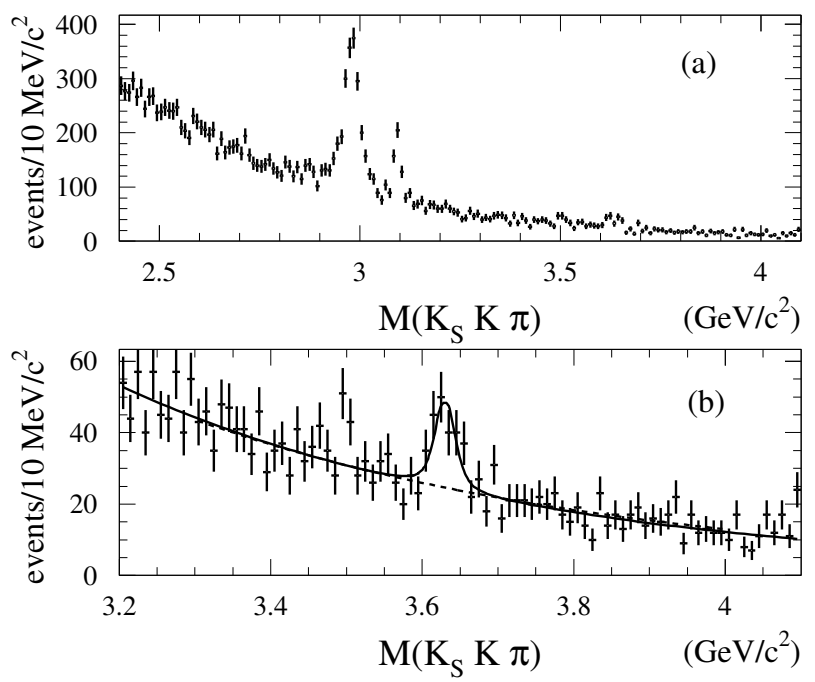

Fig. 1. The $K_{S}^{0} K^{+} \pi^{-}$invariant mass spectrum for selected events: (a) the whole region $(2.4-4.1) \mathrm{GeV} / c^{2}$; (b) detail of the $\eta_{c}(2 S)$ region, with results from the fit superimposed.

Work supported in part by the Department of Energy Contract DE-AC02-76SF00515

Stanford Linear Accelerator Center, Stanford University, Stanford, CA 94309

Presented at the International Europhysics Conference on High-Energy Physics (HEP 2003), 7/17/2003 - 7/23/2003, Aachen, Germany 


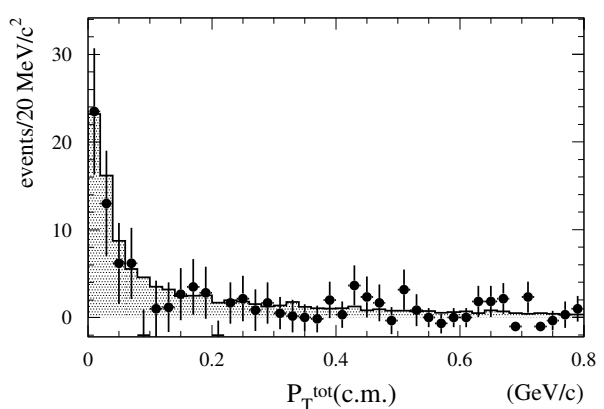

Fig. 2. Distribution of the total transverse momentum for selected events: the histogram refers to Monte Carlo simulation, the points to real data.

observed in the fitted masses on simulated events. Systematic errors account for a shift in the fitted $J / \psi$ mass on data, for uncertainties in the background-subtraction method and for differences in the resolution between real data and simulated events.

A detail of the highest part of the spectrum is shown in Fig. 1 (b): an enhancement corresponding to the $\eta_{c}(2 S)$ peak is clearly visible. A fit similar to the previous one is applied to these data, yielding

$$
\begin{gathered}
m\left(\eta_{c}(2 S)\right)=(3632.5 \pm 5.0(\text { stat }) \pm 1.8(\text { syst })) \mathrm{MeV} / c^{2}, \\
\Gamma\left(\eta_{c}(2 S)\right)=(20 \pm 10(\text { stat }) \pm 4(\text { stat })) \mathrm{MeV} / c^{2} .
\end{gathered}
$$

Also in this case a correction is applied to the mass value. Systematic errors are estimated as for the $\eta_{c}$.

The final state rules out the $J^{P}=0^{+}$assignment for this resonance. Initial state radiation production (instead of $\gamma \gamma$ ) is excluded by the angular distribution of decay products, which are concentrated in the forward hemisphere; moreover, the distribution of the total transverse momentum is peaked at 0 (Fig. 2), as expected for quasireal photons: thus the $J=1$ assignment is also ruled out. These observations strongly support the assignment of $J^{P}=0^{-}$quantum numbers, $J \geq 2$ being disfavoured for charmonium states below open-charm threshold.

\section{Observation of two narrow states decaying to $D_{s} \pi^{0}, D_{s} \pi^{0} \gamma$}

The current knowledge on the spectrum of $c \bar{s}$ mesons is limited to the ${ }^{1} S_{0}$ and ${ }^{3} S_{1}$ ground states, the $D_{s}^{+}$and $D_{s}^{*}(2112)^{+}$mesons, and to two other states, the $D_{s 1}^{+} 2536$ [9] and the $D_{s J}^{*+} 2573[10]$, for which the preferred $J^{P}$ assignments are $1^{+}$and $2^{+}$, respectively.

Another pair of states with the same $1^{+}$and $2^{+}$quantum numbers is expected to exist in the $c \bar{s}$ system. Theoretical models typically predict for these states masses between 2.4 and $2.6 \mathrm{GeV} / c^{2}$ [8]: they should decay by kaon emission and have large widths.

In this study, $D_{s}^{+}$mesons produced in continuum $e^{+} e^{-}$ interactions are reconstructed and then combined to neutral tracks. Initial selection requires the presence of two identified charged kaons, a charged pion and a photon pair reconstructing a $\pi^{0}$ with loose criteria, all originating from a common vertex. Moreover, events with candidate $D^{0} \rightarrow K^{+} K^{-}$decays are removed, and a total center-ofmass momentum above $2.5 \mathrm{GeV} / c$ is required in order to suppress background from $B$ decays.

Further cuts on the $K^{+} K^{-}$and $K^{-} \pi^{+}$invariant masses select $\phi(1020)$ and $\bar{K}^{*}(892)$ mesons as intermediate resonances. Figure 3(a) shows the resulting $K^{+} K^{-}$ $\pi^{+}$invariant mass before and after these cuts: signal and sideband regions are defined around the $D_{s}^{+}$peak.

Figure 3(c) shows the $K^{+} K^{-} \pi^{+} \pi^{0}$ invariant mass distribution for events in the $D_{s}^{+}$signal and sideband regions: a clear peak around $2320 \mathrm{MeV} / c^{2}$ is visible in the first case. The same peak is seen when selecting events from a $\pi^{0}$ signal region in the two-photon mass, whereas it is absent in $\pi^{0}$-mass sideband events (Fig. 3(b) and (d)). A fit to the $D_{s}^{+}$-sideband-subtracted $\Delta m$ distribution, where $\Delta m=m\left(D_{s}^{+} \pi^{0}\right)-m\left(D_{s}^{+}\right)$, with a Gaussian function and a linear background shape, yields

$$
\Delta m=(348.4 \pm 0.4(\text { stat }) \pm 3.0 \text { (syst) }) \mathrm{MeV} / c^{2},
$$

or $m=(2316.8 \pm 0.4$ (stat) \pm 3.0 (syst) $) \mathrm{MeV} / c^{2}$. The quoted systematic error is a very conservative estimate of resolution effects. The $D_{s J}^{*+} 2317$ notation is used for this state in virtue of the likely natural spin-parity assignment (not
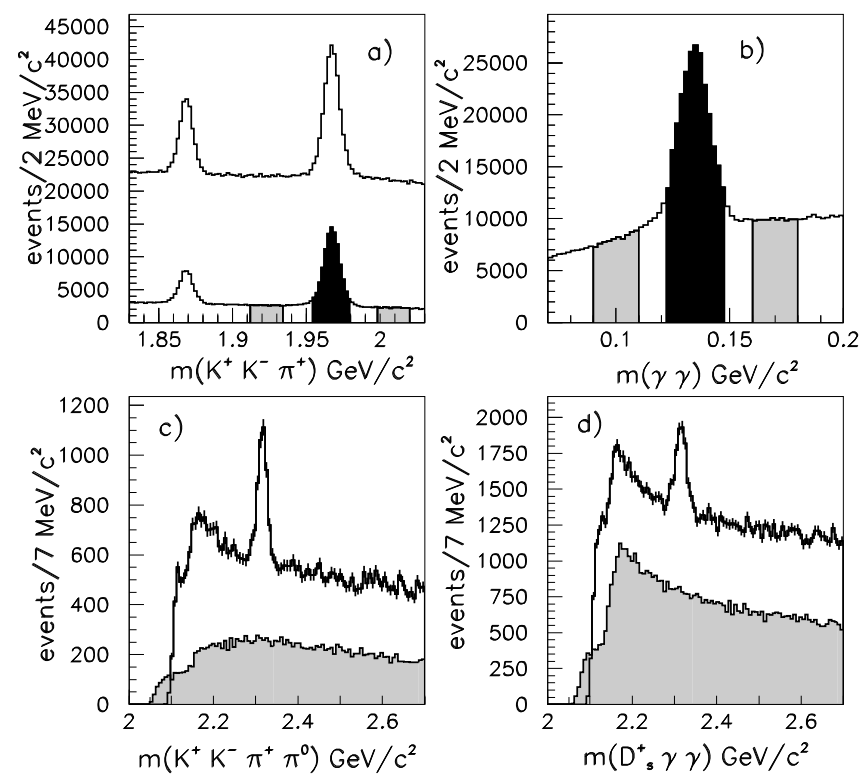

Fig. 3. (a) The $K^{+} K^{-} \pi^{+}$invariant mass distribution for all candidate events before (upper histogram) and after (lower histogram) applying requirements for $\phi \pi^{+}$and $K^{+} \bar{K}^{*}$ modes. (b) The two-photon mass distribution from $D_{s}^{+} \pi^{0}$ candidate events. In (a) and (b), signal and sideband regions are shaded. (c) The $D_{s}^{+} \pi^{0}$ mass distribution for candidates in the $D_{s}^{+}$ signal (white) and sideband (shaded) regions of (a). (d) The $D_{s}^{+} \gamma \gamma$ mass distribution candidates in the $\pi^{0}$ signal (white) and sideband (shaded) regions of (b). 

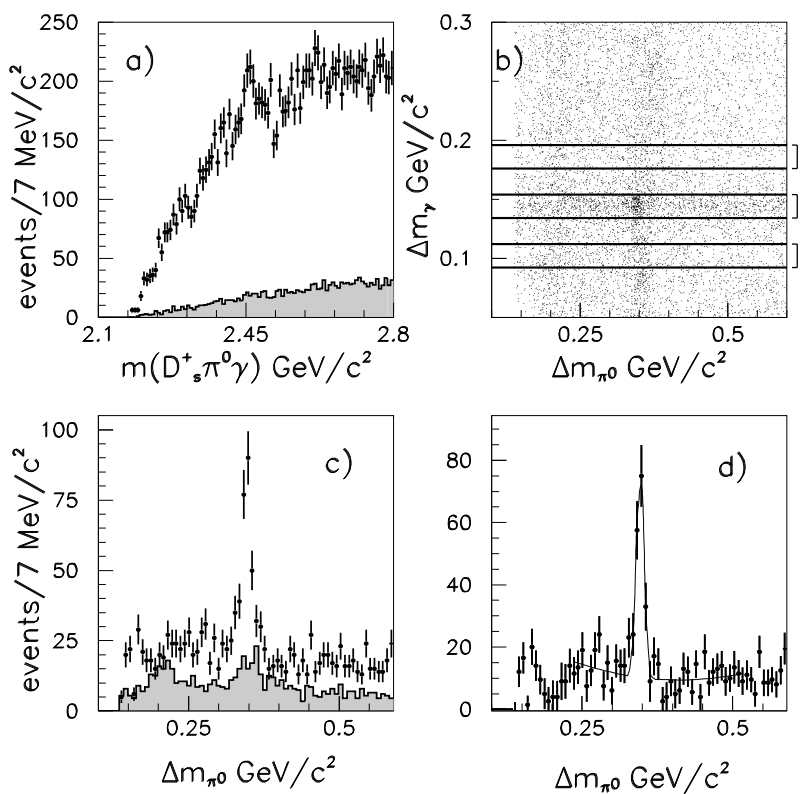

Fig. 4. (a) The mass distribution for all selected $D_{s}^{+} \pi^{0} \gamma$ combinations (top histogram) and for those in the $D_{s}^{+}$sidebands (shaded histogram). (b) The distribution of $\Delta m_{\gamma}$ versus $\Delta m_{\pi^{0}}$ for all selected combinations. Horizontal lines define three ranges in $\Delta m_{\gamma}$. (c) The $\Delta m_{\pi^{0}}$ distribution for the central $\Delta m_{\gamma}$ range (points) and for the average of the upper and lower ranges (shaded histogram). (d) Difference between the two distributions in (c), with result of the fit superimposed.

discussed here). The observed width is consistent with detector resolution. A full report of this measurement can be found in [11]. CLEO [12] and Belle [13] have confirmed the observation of this state.

A similar search is repeated in the $D_{s}^{+} \gamma, D_{s}^{+} \gamma \gamma$ and $D_{s}^{+} \pi^{0} \gamma$ channels, with or without requiring the $D_{s}^{+} \gamma$ pair to reconstruct a $D_{s 2112}^{*+}$ meson. No signal around $2320 \mathrm{MeV} /$ is observed; however, an enhancement in the $D_{s}^{+} \pi^{0} \gamma$ mass distribution is visible at a value around $2640 \mathrm{MeV} / \mathrm{c}^{2}$ (Fig. $4($ a) The same structure has been seen by the CLEO Collaboration [12], who reported observation of a new state with mass $2463 \mathrm{MeV} / \mathrm{c}^{2}$; the state has also been observed in $B$ decays by Belle [13]. It will be referred to in the following as $D_{s, J}^{+} 2457$ (because of its likely non-natural spin-parity assignment).

The fact that $m\left(D_{s_{J} 2457}^{+}\right)-m\left(D_{s 2112}^{*+}\right) \simeq m\left(D_{s J}^{*+} 2317\right)-$ $m\left(D_{s}^{+}\right) \simeq 350 \mathrm{MeV} / c^{2}$ implies that a potentially large cross-feed can be present between the $D_{s J}^{*+} 2317 \rightarrow D_{s}^{+} \pi^{0}$ and the $D_{s J 2457}^{+} \rightarrow D_{s}^{+} \pi^{0} \gamma$ channels, due to a photon being excluded from or randomly added to the combination. This can be seen in Fig. 4(b), where a scatter plot of $\Delta_{\gamma} \equiv m\left(D_{s}^{+} \gamma\right)-m\left(D_{s}^{+}\right)$vs. $\Delta_{\pi^{0}} \equiv m\left(D_{s}^{+} \pi^{0} \gamma\right)-m\left(D_{s}^{+} \gamma\right)$ is shown: the $D_{s 2112}^{*+}$ and $D_{s J 2317}^{*+}$ appear there as a horizontal and nearly vertical band, respectivley, intersecting each other at $\Delta_{\pi^{0}} \simeq 350 \mathrm{MeV} / c^{2}$. In order to separate this contribution from the true signal, two side-bands are defined in $\Delta_{\gamma}$ around the $D_{s}^{*+}{ }_{2112}$ band and the projection in $\Delta_{\pi^{0}}$ from these regions is properly subtracted from the projection in the central region (Fig. 4(c)). The resulting distribution is fitted to the sum of a Gaussian and a linear background shape, yielding for the peak value:

$$
\Delta m=(344.6 \pm 1.2 \text { (stat) } \pm 3.0 \text { (syst) }) \mathrm{MeV} / c^{2},
$$

or $m=(2457.0 \pm 1.4$ (stat) \pm 3.0 (syst) $) \mathrm{MeV} / c^{2}$, where again the systematic error is a conservative estimate of resolution effects, and the total width is consistent with detector resolution.

It is conceivable that the $D_{s J 2457}^{+} \rightarrow D_{s}^{+} \pi^{0} \gamma$ decay can proceed through $D_{s J 2457}^{+} \rightarrow D_{s 2112}^{*+} \pi^{0}$ or $D_{s J 2457}^{+} \rightarrow$ $D_{s J}^{*+} 2317 \gamma$. In order to distinguish between these two modes, data are represented in a scatter plot in the $\left(m\left(D_{s}^{+} \pi^{0}\right), m\left(D_{s}^{+} \gamma\right)\right)$ plane, where the $D_{s}^{*+} 2112$ and the $D_{s J}^{*+} 2317$ appear as a horizontal and a vertical band, respectively. The allowed phase space region for the $D_{s J 2457}^{+} \rightarrow D_{s}^{+} \pi^{0} \gamma$ decay selects a narrow area around the intersection of the two bands. A background-subtraction method similar to the one described above can be performed in both projections: the $D_{s J}^{+}{ }_{2457} \rightarrow D_{s 2112}^{*+} \pi^{0}$ decay is then expected to exhibit a narrow (broad) peak in the $m\left(D_{s}^{+} \gamma\right)\left(m\left(D_{s}^{+} \pi^{0}\right)\right)$ projection, while the opposite is expected for the $D_{s J}^{+} 2457 \rightarrow$ $D_{s J 2317}^{*+} \gamma$ decay. Comparison with distributions from Monte Carlo simulation shows that data strongly favor a dominant $D_{s J 2457}^{+} \rightarrow D_{s 2112}^{*+} \pi^{0}$ contribution.

\section{References}

1. B. Aubert et al. (BABAR Collaboration), Nucl. Inst. Meth A479, (2002) 1.

2. G. Brandenburg et al. (CLEO Collaboration), Phys. Rev. Lett. 85, (2000) 3095; F. Fang et al. (Belle Collaboration), Phys. Rev. Lett. 90, (2003) 07180; J. Z. Bai et al. (BES Collaboration), Phys. Lett. B555, (2003) 174. M. Ambrogiani et al. (Fermilab E835 Collaboration), Phys. Lett. B566, (2003) 45 .

3. K. Hagiwara et al. (Particle Data Group), Phys. Rev. D66, (2002) 010001.

4. S. K. Choi et al. (Belle Collaboration), Phys. Rev. Lett. 89, (a))(2002) 102001 .

5. Charge conjugate states are implied throughout the paper. 6. K. Abe et al. (Belle Collaboration), Phys. Rev. Lett. 89, (2002) 142001.

7. C. Edwards et al. (Crystal Ball Collaboration), Phys. Rev. Lett. 48, (1982) 70.

8. S. Godfrey and N. Isgur, Phys. Rev. D32, (1985) 189; S. Godfrey and R. Kokoski, Phys. Rev. D43, (1991) 1679; M. Di Pierro and E. Eichten, Phys. Rev. D64, (2001) 114004.

9. J. P. Alexander et al. (CLEO Collaboration), Phys. Lett. B303, (1993) 377.

10. Y. Kubota et al. (CLEO Collaboration), Phys. Rev. Lett. 72, (1994) 1972.

11. B. Aubert et al. (BABAR Collaboration), Phys. Rev. Lett. 90, (2003) 90242001.

12. D. Benson et al. (CLEO Collaboration), hep-ex/0305100, now published in Phys. Rev. D68, 032002 (2003).

13. R. Seuster et al. (Belle Collaboration), hep-ex/0307041, contributed to this Conference; now superseded by hepex/0308019. 